\title{
Status of the Polarized Nonlinear Inverse Compton Scattering Experiment at UCLA
}

\author{
O. Williams ${ }^{*}$, A. Doyuran ${ }^{*}$, R.J. England ${ }^{*}$, C. Joshi ${ }^{\dagger}$, J.B. Rosenzweig ${ }^{*}$, \\ S. Tochitsky ${ }^{\dagger}$, G. Travish ${ }^{*}$ \\ ${ }^{*}$ Department of Physics and Astronomy, University of California, Los Angeles \\ ${ }^{\dagger}$ Department of Electrical Engineering, University of California, Los Angeles, \\ Los Angeles, CA 90095, USA
}

\begin{abstract}
An Inverse Compton Scattering (ICS) experiment investigating the polarized harmonic production in the nonlinear regime has begun which will utilize the existing terawatt $\mathrm{CO}_{2}$ laser system and $15 \mathrm{MeV}$ photoinjector in the Neptune Laboratory at UCLA. A major motivation for a source of high brightness polarized x-rays is the production of polarized positrons for use in future linear collider experiments. We report on the experimental set-up and status.
\end{abstract}

Keywords: Compton scattering, laser, X-ray, photon, harmonics

PACS: $52.59 . \mathrm{Rz}, 41.75 . \mathrm{Fr}, 52.59 . \mathrm{Sa}, 41.60 . \mathrm{Bq}$

\section{INTRODUCTION}

The production of third-generation light source quality x-rays using low energy electron beams as well as the possibility of using it as a source of polarized positrons in high energy physics experiments has spurred much interest in inverse Compton scattering (ICS) $[1,2]$. Of particular effort is determining the laser and electron beam parameters necessary to obtain maximum scattered photon flux. This search has led to different approaches such as increased interaction cross-section (e.g. smaller spot sizes, increased laser power, and longer laser wavelength) or increased interaction length by guiding (e.g. in plasma) [R. Yoder, these Proceedings]. The scenarios for increased interaction cross-section have led to a condition where nonlinear scattering occurs and very high laser fields induce relativistic transverse motion of the electrons leading to higher harmonic generation. To reliably use inverse Compton scattering as a high brightness x-ray source requires an understanding of the nonlinear properties of the scattering process during high laser intensities (where the laser vector potential, $\left.a_{0} \geq 1\right)$.

The observed angular distribution of the $2^{\text {nd }}$ harmonic in a $180^{\circ}$ interaction for different laser polarizations was recently reported [3] and presented in this conference [I. Pogorelsky, these Proceedings]. The spectral broadening of the ICS spectrum associated with the ponderomotive scattering of the electrons in the high intensity laser field is still uncharacterized and it has yet to be shown whether the laser polarization is transferred to the scattered photons during the nonlinear interaction.

\footnotetext{
CP877, Advanced Accelerator Concepts: $12^{\text {th }}$ Workshop, edited by M. Conde and C. Eyberger

(C) 2006 American Institute of Physics 978-0-7354-0378-9/06/\$23.00
} 
Another unexplored phenomenon associated with nonlinear ICS is the red-shift of the fundamental as $\mathrm{a}_{\mathrm{o}}$ increases, as is seen in the expression,

$$
E_{x}=\frac{h c}{\lambda_{x}}=\frac{4 \gamma^{2}}{\lambda_{L}}\left(1+\frac{a_{o}^{2}}{2}+\gamma^{2} \theta^{2}\right)^{-1}
$$

where $\lambda_{L}$ is the wavelength of the interaction laser and $\theta$ is the observation angle. Experimental observation of this effect created by the intense laser field strongly deflecting the electrons transversely is of interest for the physics and its potential as a method of source tuning. As can be seen, there exists much to be learned about the nonlinear scattering of a high intensity laser pulse off a relativistic electron beam and further research should give more insight into the limitations of using a laser or other electromagnetic field as an undulator for the production of high brightness radiation.

The Neptune Laboratory at UCLA houses a terawatt-class $\mathrm{CO}_{2}$ laser system capable of achieving $a_{0}=1$ [4]. Very strong focusing is therefore required at the interaction point (IP) for both the laser and electron beam. In order to focus the $14 \mathrm{MeV}, 300 \mathrm{pC}$, low emittance beam produced by the 1.6 cell S-Band photoinjector and linac, the development of an array of permanent magnet quadrupoles (PMQ's) was necessary. The strong diffraction due to the short laser focal length and small aperture of the PMQ's did not permit counter-propagation of the two beams and necessitated a transverse $\left(90^{\circ}\right)$ scattering geometry. Although this scattering angle produces fewer ICS photons than in the head-on case, ultra-short (sub-picosecond) pulses can be created if the parameters are chosen correctly. Laser polarization along the axis of electron beam propagation is also possible, making this an interesting interaction geometry which has yet to be investigated.

\section{EXPERIMENTAL SET-UP}

The layout of the interaction region is shown in Fig. 1. In order to maximize photon flux under nonlinear scattering conditions, strong focusing is required of both beams. Conventional electromagnet quads were not employed due to the dispersion,

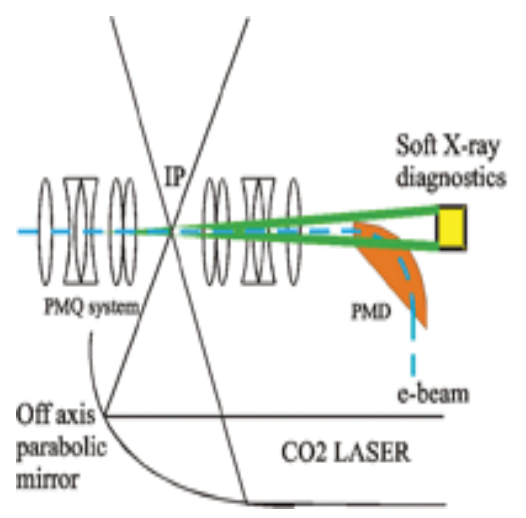

FIGURE 1. Schematic drawing of the interaction region. 
TABLE 1. Design Electron and Laser Beam Parameters

\begin{tabular}{l|c} 
Parameter & Value \\
\hline Electron Beam Energy & $14 \mathrm{MeV}$ \\
\hline Beam Emittance (norm. RMS) & $5 \mu \mathrm{m}$ \\
\hline Electron Beam Spot size (RMS) & $25 \mu \mathrm{m}$ \\
\hline Beam Charge & $300 \mathrm{pC}$ \\
\hline Bunch Length (RMS) & $4 \mathrm{ps}$ \\
\hline Laser Beam radius at IP (RMS) & $25 \mu \mathrm{m}$ \\
\hline $\mathrm{CO}_{2}$ Laser Wavelength & $10.6 \mu \mathrm{m}$ \\
\hline $\mathrm{CO}_{2}$ Laser Rayleigh Range & $0.75 \mathrm{~mm}$ \\
\hline $\mathrm{CO}_{2}$ Laser Power & $500 \mathrm{GW}$ \\
\hline $\mathrm{CO}_{2}$ Laser Pulse Length & $200 \mathrm{ps}$ \\
\hline
\end{tabular}

chromatic aberrations, and strong space charge forces associated with focusing the lower energy electron bunch [5]. To achieve the small beam sizes, PMQ's were developed with a gradient of $\sim 115 \mathrm{~T} / \mathrm{m}$. Five total PMQ's are arranged in a modified triplet configuration (F-DD-FF) with only $1.5 \mathrm{~cm}$ to the minimum beam waist (IP), followed by a symmetric triplet for recollimation. Both PMQ assemblies are manually adjustable with respect to the IP and remotely adjustable as a unit to allow scanning of the waist along the beam axis.

It can be seen in the expression for the laser vector potential, $a_{0}=\lambda_{L} e E_{L} / 2 \pi m_{e} c^{2}$, where $e$ is the charge of an electron, $E_{L}$ is the energy of the laser pulse, and $m_{e}$ is the mass of an electron, that longer wavelength light, $\lambda_{L}$, makes $\mathrm{a}_{\mathrm{o}}$ large, giving an advantage to $\mathrm{CO}_{2}$ lasers. However, a high intensity spot is still required at the IP to achieve $\mathrm{a}_{\mathrm{o}}=1$ and for $500 \mathrm{GW}$ of $10.6 \mu \mathrm{m}$ light, this necessitates a $25 \mu \mathrm{m}$ spot (RMS). This strong focusing is accomplished by a 5" copper off-axis parabolic mirror with an F\# of 1.5 oriented $90^{\circ}$ with respect to the beam axis, resulting in orthogonal scattering with the electron beam. The design parameters for the electron and laser beams are shown in Table 1.

Due to the low energy electron beam, the scattered photons are emitted into a rather large cone of half angle $\left(\theta_{\mathrm{X}} \sim 1 / 2 \gamma\right) \theta_{\mathrm{X}}=15 \mathrm{mrad}$ (see Table 2 for more scattered photon parameters). This large angle imposed strong space constraints on the experimental layout. In order to place the x-ray diagnostics (i.e. X-ray CCD camera) as close to the source as possible to fully image any off-axis harmonics, the electron beam needed to

TABLE 2. Calculated Scattered Photon Parameters

\begin{tabular}{l|c|c} 
Parameter & Head-on & Transverse \\
\hline Scattered photon wavelength & $5.3 \mathrm{~nm}$ & $10.7 \mathrm{~nm}$ \\
\hline Scattered photon energy & $235 \mathrm{eV}$ & $118 \mathrm{eV}$ \\
\hline Scattered photon pulse duration $(\mathrm{FWHM})$ & $10 \mathrm{ps}$ & $10 \mathrm{ps}$ \\
\hline Interaction time & $5 \mathrm{ps}$ & $0.33 \mathrm{ps}$ \\
\hline \# of periods electrons see & 283 & 10 \\
\hline \# of photons emitted per electron & 3.34 & 0.11 \\
\hline Total \# of photons & $6.3 \times 10^{9}$ & $2 \times 10^{8}$ \\
\hline Half Opening Angle $\left(\theta_{\mathrm{X}}\right)$ & $2.7 \mathrm{mrad}$ & $15 \mathrm{mrad}$ \\
\hline Bandwidth & $0.35 \%$ & $10 \%$ \\
\hline
\end{tabular}


be bent and dumped immediately following recollimation, requiring a compact permanent magnet dipole (PMD).

\section{IP Diagnostics}

The spatial overlap and synchronicity of arrival at the IP between the electron and laser beam is critical to obtain sufficient flux of scattered photons. The high density of charge in the $25 \mu \mathrm{m}$ electron spot at the IP makes traditional YAG or phosphor imaging less desirable due to limited spatial resolution [6]. Therefore, we chose to image the electron beam using optical transition radiation (OTR). The difficulty of imaging a $90^{\circ}$ interaction is also complicated by the compactness of the experiment. The distance between the focusing and recollimating quads is only $3 \mathrm{~cm}$ and must accommodate the diffracting laser beam. The transport of light at the IP intended for alignment diagnostics therefore required an unconventional layout. This prompted the development of a highly reflective, sharp-edged, aluminum pyramid used to simultaneously image the two beams as seen in Fig. 2.

The $10.6 \mu \mathrm{m}$ light, not lying in the visible spectrum, requires the application of a thin layer of micron-sized graphite on the laser imaging surface of the pyramid which "sparks" when hit by the laser, allowing for both beams to be overlapped along the pyramid's edge as imaged by a vertically mounted CCD camera. The two beams must also be synchronized in time at the IP. Temporal overlap is accomplished through a Germanium crystal acting as a "gate". When the beam electrons are incident on the crystal oriented at $45^{\circ}$, a semiconductor plasma is formed which is opaque to the 10.6 $\mu \mathrm{m}$ laser light. By measuring the transmitted intensity of the laser, the relative timing between the beams is revealed and allows for tuning of the arrival time at the IP [7].

\section{X-ray Diagnostics}

Two types of x-ray imaging devices are planned for use in the experiment, a soft $\mathrm{x}$ ray CCD camera (on loan from the ANL APS) and a microchannel plate (MCP) detector. The X-ray camera will be used to image primarily the beam axis with good

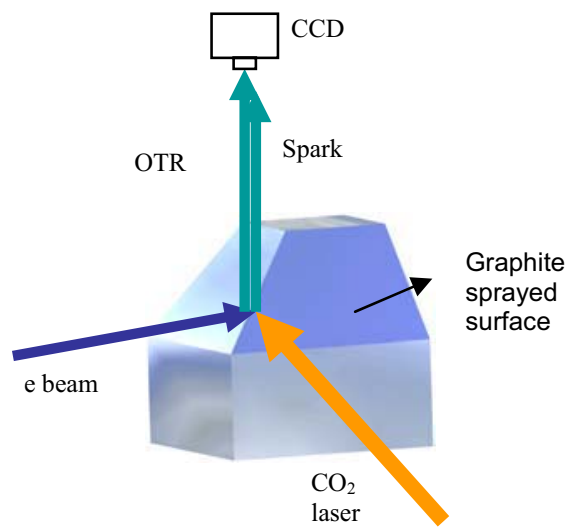

FIGURE 2. The IP imaging diagnostic "pyramid". 
resolution. Because the $\mathrm{x}$-ray source is diverging so strongly and the $\mathrm{x}$-ray camera has an imaging area of only $12.3 \times 12.3 \mathrm{~mm}^{2}$, the experiment has been designed to allow for a reentrant housing such that the camera can be inserted into the vacuum chamber, closer to the source.

The MCP detector has a $40 \mathrm{~mm}$ diameter imaging area and is mounted further from the source than the X-ray camera, yet still has $75 \%$ more field of view. After being cross-calibrated against the x-ray camera, the MCP will be used to better image the beam off-axis, particularly the expected higher harmonics.

As the magnitude of the laser field at the interaction point is increased the creation of harmonics up to $n=3$ and $n=4$ occurs. The small energy range $(\sim 100-500 \mathrm{eV})$ within which the harmonics are being produced make it difficult to use filters as attenuators to cut-off the fundamental and lower harmonics. Instead, we will attempt to use the strong absorption of the Carbon K-edge at $284 \mathrm{eV}$ to differentiate between the harmonics. The transmission curve for $100-500 \mathrm{eV}$ photons through one micron of Carbon is shown in Figure 3. Both the $2^{\text {nd }}$ and $3^{\text {rd }}$ harmonics can occur at this energy if the electron beam energy is slightly reduced to $\sim 12.6 \mathrm{MeV}$ and the laser power is relaxed to $75 \mathrm{GW}$ (in the case of the $2^{\text {nd }}$ harmonic, resulting in $\mathrm{a}_{\mathrm{o}}=0.39$ but still creating sufficiently measurable flux). As mentioned earlier, the red-shift of the fundamental with increasing laser field is an informative demonstration of the physics involved in the nonlinear interaction of electrons in an intense electromagnetic field and which offers another method of tuning the X-ray source. We wish to observe this shift in photon energy (far downstream and on-axis, so $\theta=0$ in Eq. 1) by varying the incident laser power while keeping all other parameters fixed. It can be seen in Figs. 4(a) and 4(b) that by spanning the range, $P_{L}=75-500 \mathrm{GW}$, the values of the laser vector potential span, $a_{0}=0.1-1$. Correspondingly, the scattered photon energy for $\mathrm{n}=1$ is increasingly red-shifted producing a range of $\mathrm{E}_{\mathrm{X}}=150-100 \mathrm{eV}$ for $\mathrm{a}_{\mathrm{o}}=0.1-1$, respectively.

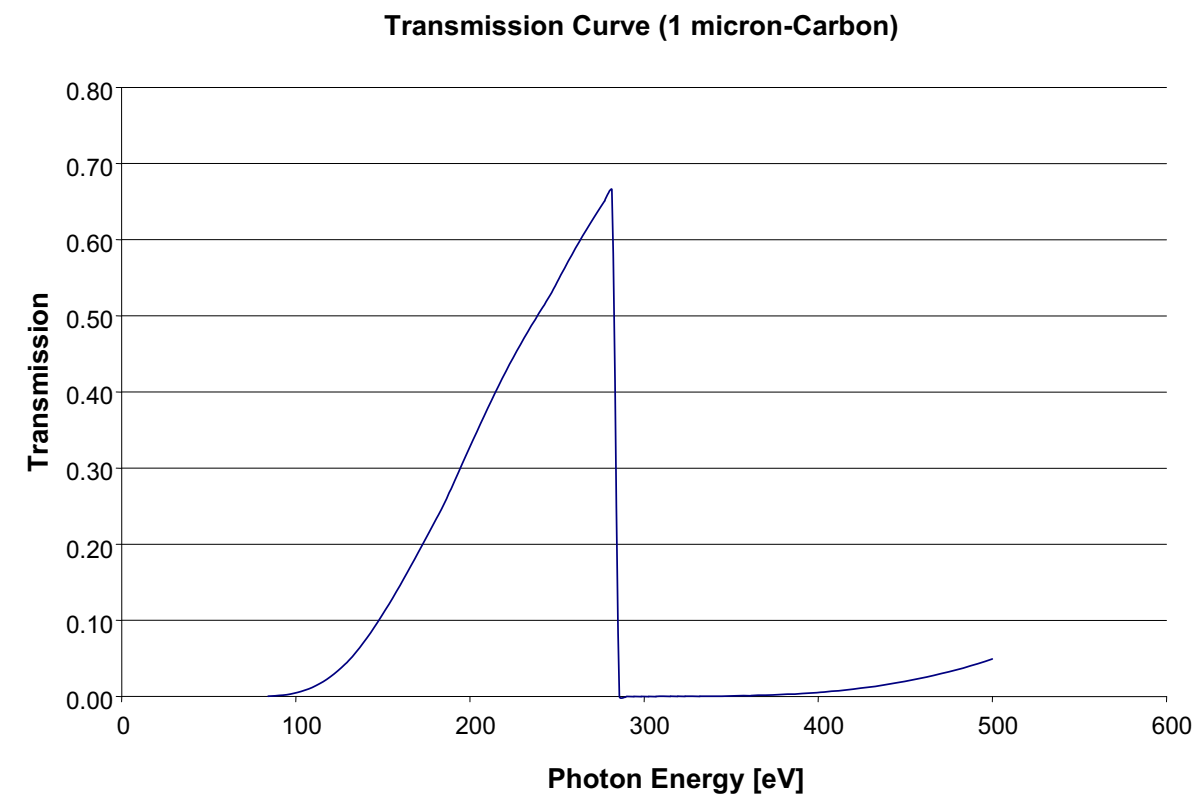

FIGURE 3. Photon transmission through a $1 \mu \mathrm{m}$ Carbon filter. Strong absorption at the Carbon Kedge of $284 \mathrm{eV}$ can be seen. Data obtained through Center for X-ray Optics (CXRO) website. 


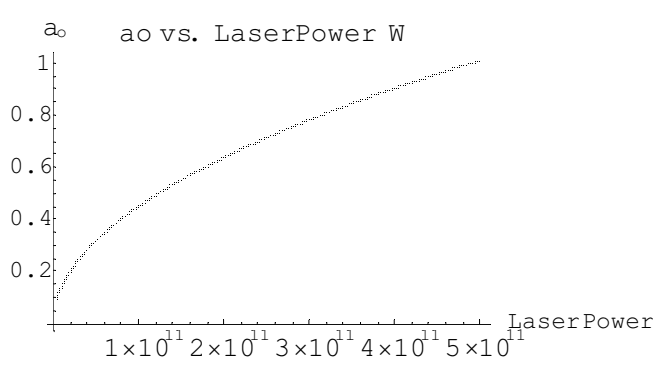

(a)

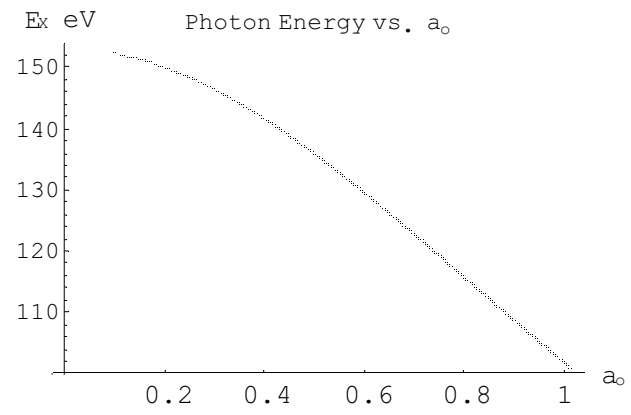

(b)

FIGURE 4. The effect of the laser power on the normalized vector potential (a) and the expected corresponding energy red-shift of the scattered photons within the fundamental (b).

Due to the inherent linear response to photon energy of a CCD detector $\left(3.65 \mathrm{eV} / \mathrm{e}^{-}-\right.$ hole pair), the possibility of using the soft x-ray camera as a non-dispersive energy spectrometer to resolve the red-shift has been investigated. This method of energy measurement requires very low background levels (produced by the laser beam and electron bremsstrahlung) and single photon counting of the scattered photons. Careful analysis using modern imaging software will reveal the charge accumulated (and thus incident photon energy) in each pixel during an exposure to the ICS photon pulse.

Polarization measurements in the EUV/soft x-ray region are fairly difficult and in order to adequately show that the laser polarization is imprinted onto the scattered photons during the nonlinear interaction it has been proposed to use transmissive multilayer optics. Thus far this appears to be the simplest method for soft x-ray polarimetry although obtaining such optics has yet to be discussed.

\section{CALCULATION TOOL}

The experiment is well-coupled with a strong theoretical framework on the expected double differential spectrum (DDS). A Mathematica routine has been developed which predicts the angular and frequency spectra for the scattered photons at different scattering angles and laser polarizations and pulse shapes. A more detailed discussion on the expected distributions of higher harmonics can be found in Ref. [8].

\section{STATUS OF THE EXPERIMENT}

Electron and laser beam background measurements were taken separately as seen on the x-ray CCD camera. Analysis of the electron beam background reveals a decaying exponential indicative of solid target bremsstrahlung which illuminates $\sim 1.6 \%$ of the total pixel area. Laser background is created by beam halo in the nonGaussian, $100 \mathrm{~J}$ beam scraping on the yokes of the PMQ's and on the exit port of the vacuum chamber. Teflon apertures slightly smaller than the beam have been placed in these locations to prevent laser reflection back upstream. A drawback is the creation of low energy, broadband radiation, much of which exists in the visible and causes the Teflon to "glow". The average increase in signal background in comparison to the 
dark bunker is 90 ADU (analog-to-digital units). To combat this, a light-reducing baffle is being constructed which will help in capturing and absorbing any stray light making it down the pipe toward the camera. Considering the camera sensitivity of $\sim 45 \%$ QE for a $100 \mathrm{eV}$ photon and the current gain setting (2 $\left.\mathrm{e}^{-} / \mathrm{ADU}\right)$, we can expect a scattered photon signal of 6 ADU. While this can be resolved by the 16-bit camera, we wish to reduce the background to minimal levels before attempting to see ICS photons.

\section{REFERENCES}

1. W. Thomlinson, Nucl. Instrum. Methods A 319, 295 (1992).

2. T. Omori et al., Nucl. Instrum. Methods A 500, 232 (2003).

3. M. Babzien et al., Phys Rev Letters 96, 054802 (2006).

4. S. Tochitsky et al., Proc. of AAC 2004, AIP Conf. Proc. 737, 663 (2004).

5. J. Lim et al., Phys Rev ST Accel. Beams 8, 072401 (2005).

6. A. Murokh et al., PAC 2001 Proceedings, p. 1333 (2001).

7. S. Tochitsky et al., Physics of Plasmas 11(5), 2875 (2004).

8. G.A. Krafft et al., Phys Rev E 72, 056502 (2005). 